\title{
Accelerating optical reporting for conformation of tyrosine kinase inhibitors in solutions
}

\author{
Feng Wang ${ }^{1}$ and Vladislav Vasilyev ${ }^{2}$ \\ ${ }^{1}$ Swinburne University of Technology \\ ${ }^{2}$ Australian National University
}

March 11, 2021

\begin{abstract}
It has been a challenge in automated analysis of medical and chemical knowledge to extract represent quantitative structureactivity relationship (QSAR) using intelligent computing in drug discovery. One of many domain-specific bottlenecks in drug discovery is robust conformation search in three-dimensional (3D) space for flexible drug candidates. The process involves researchers and machines working together to achieve their own strengths for greater outcome. The present study has been developing a method for conformational sampling conformers in the class of 4-anilinoquinazoline derivatives for epidermal growth factor receptor (EGFR) tyrosine kinases inhibitors (TKIs). We use AG-1478 to demonstrate how the new intelligent computing method helps to quantum mechanically determine 22 target drug conformer clusters and their properties from conformational sampling, based on density functional theory (DFT) method, time-dependent (TD)-DFT in solvents and clustering analysis (CA). The UV-vis spectra of the preferred conformers agree well with earlier experimental measurements in which the conformer dependent UV-Vis spectral shift of AG-1478 can be as large as approximately $15 \mathrm{~nm}$. We are further developing this method to study and design new 4-anilinoquinazoline derivatives of EGFR TKIs.
\end{abstract}

\section{Hosted file}

TKIs_EGFR_IJQC.pdf available at https://authorea.com/users/400934/articles/513243accelerating-optical-reporting-for-conformation-of-tyrosine-kinase-inhibitors-insolutions 